\title{
Choosing between organisational \& technical safety: a practical approach for industry
}

\author{
Johan Gort ${ }^{1}$, Johan Reinders ${ }^{2}$ and Sander L.J. Zwanikken ${ }^{1}$, \\ ${ }^{1}$ TNO Work \& Employment, Hoofddorp, The Netherlands \\ ${ }^{2}$ TNO Environment, Energy and Process Innovation, Apeldoorn, The Netherlands
}

\begin{abstract}
For several years it has been tried to make a connection between organisational and technical safety. Researchers have successfully integrated human factors components in accident investigation tools [1] or have developed an elaborate model in which organisational and technical elements are coupled together [2,3]. These approaches are useful for either the organisation that wishes to learn more from accidents or for researchers who are studying connections between two separate worlds of scientific subjects. Management of industries however likes to know firstly its actual safety performance and secondly in what area of safety money should be spent $[4,5]$. Neither of the tools mentioned is sufficiently equipped to give these answers. TNO has combined a technical risk assessment with an organisational safety assessment. The result is the use of a combination of instruments, techniques and methods that enforce each other and give a company good insight in what the state of safety management is. The outcomes of the different surveys lead to a solid comparison of technological and organisational measures to be taken and serve as a basis for further decision making. The method is tested in a pilot study in a chemical production site. This paper describes the developed methods for organisational safety and the use of it by the case company.
\end{abstract}

\section{Introduction}

In 2002 TNO started a project in which the know-how of a number of TNO institutes was combined and which was aimed at integrating technical safety analyses with an organisational safety approach, with the ultimate goal to enable companies to make balanced decisions regarding (safety) investments. The technical studies were based on the 'Layer of Protection Analysis' (LOPA), a tool to be further developed for the 
combined safety assessment. TNO Work \& Employment adapted the organisational safety analyses in such a way that they could be incorporated inthe more technical and (semi)quantitative safety-analyses. This leads to new insight in the most effective way for a company to decide which safety measures should be implemented. Eventually, it should lead to a decision making tool for management. The tool helps companies to make integral and rational decisions around safety and business.

This paper focusses on the possibilities to incorporate organisational analyses into the technical safety-analyses. We describe the individual instruments and the way we combine them. At the congress we will present the results of the case-study and the way companies can integrate these analysis in "normal" business.

\section{Method and used instruments}

\subsection{Introduction}

The central tool used forthe technical risk analyses of the chemical process under study was the Layer Of Protection Analysis (LOPA). This method is a semiquantitative tool for analysing and assessing risks and is based on the identification of potential accident scenarios. A particular scenario will only take place if certain undesired initiating events (e.g. overpressure, operator failure) occur and if protective layers fail or are absent. There are various protection layers in a chemical process, all of which help to prevent an accident scenario to develop.

New in this instrument is the Safety Quality Factor (SQF). The SQF is based on qualitative analysis of the safety organisation and the maintenance organisation. The SQF influences the probability of occurrence of the accident scenarios by reducing or increasing the frequency of initiating events and/or one or more protection layers.To determine the SQF an extensive organisation survey is performed.

For the organisational analysis we combine three instruments:

1. Quick scan OHSAS 18001

2. Tripod delta ${ }^{\circledR}$ analysis

3. Quick scan safety culture.

These three methods analyse different parts of a organisation: the OHSAS audit focuses on the safety management system, the Tripod ${ }^{\circledR}$ Delta analyses the level of control the management has on the working environment / the effectiveness of the safety management system (described by Basic Risk Factors), and the quick scan safety culture is developed to analyse the assumptions about the safety culture that management has and for comparison these with evidence of the existing safety culture found at the work floor. The overall results lead to insights of the good and weaker parts of the safety management organisation and give priorities for improvements. Besides that it leads to an overall judgement of the safety management organisation, expressed by the Safety Quality Factor. 
The overall analysis leads to knowledge of quantitative risks, the technical safety level of the company and the quality of the safety organisation. Calculating the investments for the different measures and the results that will be achieved with them, leads to the situation that management have insight in the cost-effectiveness of several options of investment. A management-services tool helps the company to make more integral and more rational decisions around safety and business and to monitor the safety improvement by the use of safety performance indicators. This tool helps companies to improve safety on an effective and efficient way. in more detail.

In the next two paragraphs we describe the different analysing instruments

\subsection{Layer of protection analysis (LOPA)}

LOPA was developed in the 1980's to facilitate decision-making and answering key questions using a rational, objective and risk-based approach [6]. The key questions are e.g. How safe is safe enough? How many protection layers are necessary? How much risk reduction will each layer provide?

LOPA evaluates the individual protection layers as proposed or as present in a given process for their effectiveness and subsequently compared against risk tolerance criteria. In this way the method provides a tool to compare required safety levels with safety measures.

LOPA is a simplified form of risk assessment as typically "order of magnitude" categories for initiating event frequencies, consequence severity and the likelihood of failure of independent protection layers (IPLs) are taken into account. Using this information, the risk of a scenario is assessed. The method thus falls in between qualitative methods like HAZOP, What-if or FMEA and a quantitative method like QRA. LOPA is thus not a fully quantitative risk assessment approach. It is a simplified method to assess the value of protection layers for a well-defined accident scenario. LOPA does not suggest which IPLs to add or which design to choose. It merely assists in judging between alternatives for risk mitigation

\subsubsection{The layers of protection}

Typically, the following types of IPLs are defined:

1. Process design,

2. Basic process control system (BPCS),

3. Critical alarms and human interventions,

4. Safety instrumented functions (SIF), SIS or ESD,

5. Physical protection (relief devices),

6. Post release physical protection (walls, dikes),

7. Plant emergency response,

8. Community emergency response.

Layers 1 to 4 are preventive measures. These measures are aimed at the prevention of a so-called Loss Of Containment (LOC). In terms of risk such a measure is considered to reduce the probability of an LOC. Layers 5 to 8 are mitigation measures. These measures are aimed at minimising the consequences. In terms of 
risk, a mitigating measure is considered to reduce the effect or consequence. The characteristics of the independent safety layers are that each safety layer is totally independent of other safety layers; is not affected by failure of other layers; must have an acceptable reliability; must be approved to company policy and procedures; must meet proper equipment classification; must be a non-control alternative; may require diverse hardware and software packages; may be an administrative procedure.

To prevent the occurrence of a scenario (or the consequences of an initiating event) only one layer of protection is required. The essence of LOPA is based on the fact that both preventive and mitigating measures have a probability of failure on demand (PFD). The primary purpose of LOPA is to determine if there are sufficient layers of protection against the scenario to balance the safety measures with the required safety levels. If insufficient safety measures are present, additional layers may be added. Or, the other way around, if too many layers are present, layers may be deleted (safety critical equipment or instrumentation). For a scenario to occur, all IPLs should fail.

\subsubsection{Scenario}

Scenario development is an essential step in the LOPA analysis. Scenarios are developed by a team of experts on the particular installation or process, often making use of existing safety studies like e.g. Hazard and Operability Analysis (HAZOP's) or Failure Mode and Effect Analysis (FMEA's) The result of such an analysis is a list of accident scenario's, with associated frequencies of occurrence. An accident scenario is a single cause - consequence chain

\subsubsection{LOPA Target Factor and Safety Gap}

To find out if a particular scenario frequency is acceptable acceptability criteria need to set up. These criteria are called the LOPA Target factors (LTF). LTFs can e.g. be created using a risk matrix. For the considered consequences (typically five or so) a maximum allowable frequency is defined. This is called the LOPA Target Factor (LTF) Frequencies of LOPA scenarios that exceed the LTF are not acceptable. The difference between the scenario frequency and the LTF is called the Safety Gap. The process is safe enough when there is no safety gap (left).

\subsection{Organisational instruments}

Each method used for the organisational analysis has a different focus. By factfinding on different organisational levels we triangulate our findings as a validation step. The three different instruments for the organisational analysis are described in the following paragraphs.

\subsubsection{The Safety Culture Quick Scan}

The Quick Scan Safety Culture is a new instrument that has been tested in the case study. The quick scan focuses on perceived and actual values and believes on safety of the organisation and results in a short list of possible improvements. 
We start with a management meeting in which 10 pre-selected safety management problems are ranked. Pre-selection is based on literature data and on our experience from earlier research. In the next steps we try to find evidence in the company for the top three of perceived problems by document analysis, interviews and observations. Also the employees fill in a questionnaire about the safety culture. The data of the safety culture questionnaire are statistically analysed. Subsequently these results are verified in interviews.

At the end we know which perceived problems are truly present in the company, and also if there are any other major management problems with safety culture.

\subsubsection{The OSHAS 18001 quick scan}

The OSHAS 18001 quick scan is mainly an audit method based on the OHSAS 18001. OSHAS 18001 contains requirements for safety management systems based on the ISO 9000 series of quality management [7]. In the case study we try to assess:

- The level of description or the actual presence of the management system, written procedures, instructions etc.

- To what degree the written management system is implemented

- The way management evaluate the quality of the system.

- The way the company improves the management system and the actual safety and health of the working place.

\subsubsection{Tripod Delta}

Tripod delta is a model developed by the University of Leiden and Manchester (Groeneweg 1998, [8]) and is used to measure latent failures in the working environment that can cause human error. Accidents are often attributed to human error, but accident analysis points out that contributing causes of accidents can be traced back to elements of the working environment and eventually management decisions. These controllable parameters are called the Basis Risk Factors (BRF's): Design (DE), Hardware (HW), Maintenance management (MM), Housekeeping (HK), Error enforcing conditions (EC), Procedures (PR), Training (TR), Communication (CO), Incompatible goals (IG), Organisation (OR), Defences (DE). The Tripod ${ }^{\circledR}$ Delta survey is a checklist-based survey and evaluates actual performance of an organisation. The results of the survey is a quantitative profile supported by a qualitative interpretation of the findings. This gives management information on how to eliminate the structural weaknesses in the organisation, how to consolidate the strong points and how to set priorities in action planning.

\section{4 The safety quality factor}

Many activities with respect to safety work in an indirect way. Training, for example, will affect the IPL 'critical alarms, human intervention'. It may also reduce the frequency of certain initiating events (e.g. operator failure). However, the training activity itself will never prevent a scenario from occurring. Likewise, maintenance will affect the failure frequency of many IPLs, but the activity itself won't stop a scenario from developing. The quality of such safety (related) activities can be 
determined by the organisational review methods described here. By calculating the outcome and combining the results of the different surveys, it is possible to determine the quality of organisational safety. This is semi-quantified in a so-called Safety Quality Factor (SQF). This SQF can be included in the assessment of the failure frequency of an IPL or the frequency of initiating events as part of the determined scenarios.

\subsection{The management tool}

All results are discussed in a meeting with the management of the company. Goal of this first meeting is to commit management to the findings and to set priorities. In a second meeting we focus on these priorities, trying to find solutions, resulting in concrete actions. We also formulate performance indicators to be included in a Balanced Score Card of the company.

\section{Results and follow-up}

\subsection{Safety culture QS}

In a start up meeting the management of the chemical company selected the following safety management problems:

- There are differences in safe behaviour and safety culture between teams in the organisations

- Middle management is not a good enough example in safe behaviour

- Top management doesn't know the actual safety performance and behaviour on the work floor

- Safety is not always as important as production

- We do things first, we think afterwards.

All managers accept not all management problems mentioned above but we decided to investigate these five problems.

We found that there were no significant differences between the different teams. The management opinion is based on problems with only one team leader in the past. These problems are solved now, as are the differences between the teams.

We found that middle managers set a good example in safe behaviour. However supervision and correcting employees is not implemented consistently. Management does know the actual safety performance in global terms. There is less knowledge of daily safety and safe behaviour at the work floor.

Although in some instances production was reported to be more important than safety, we think this is more the exception than the rule and that normally safety is as important as production in this company.

\subsection{QS OHSAS audit}

The quick scan OHSAS audit resulted in a list of non-conformities when compared to the norm document. The main nonconformities are: 
- Safety policy does not include commitment to continuous improvement and does not include the intention to follow legislation and regulations

- Maintenance management and safety procedures are insufficiently implemented

- The inspection system is insufficiently implemented in several places.

The results of the quick scan are translated in a figure per element of the OHSAS 18001. We use the following scoring system for each element: $1=$ should be started, $2=$ Intention available, $3=$ Procedures available, $4=$ Procedures implemented, 5= Monitoring and controlling finds place.

\subsection{Tripod Delta}

The Tripod Delta survey resulted in a list of findings categorised in basic risk factors (BRF), the latent failures management should control. Each BRF has relative strong points (scores of $90 \%$ or more on the questions) and relative weak points (score of less than $70 \%$ on the questions). These are presented in a separate overview. The main weak points were:

- Planned maintenance work has been postponed during the last three months because the costs were too high (BRF Incompatible goals, DO).

- Not every supervisor can be considered competent for his work (BRF Training \& competencies, TR).

- It has happened that a repair has been done only partially during the last three months (BRF Maintenance management, $\mathrm{OH}$ ).

- Safety meetings have not resulted in obvious improvements in the working area during the last three months (BRF Communication, $\mathrm{CO}$ ).

The following profile has been made from the case company (see figure 3).

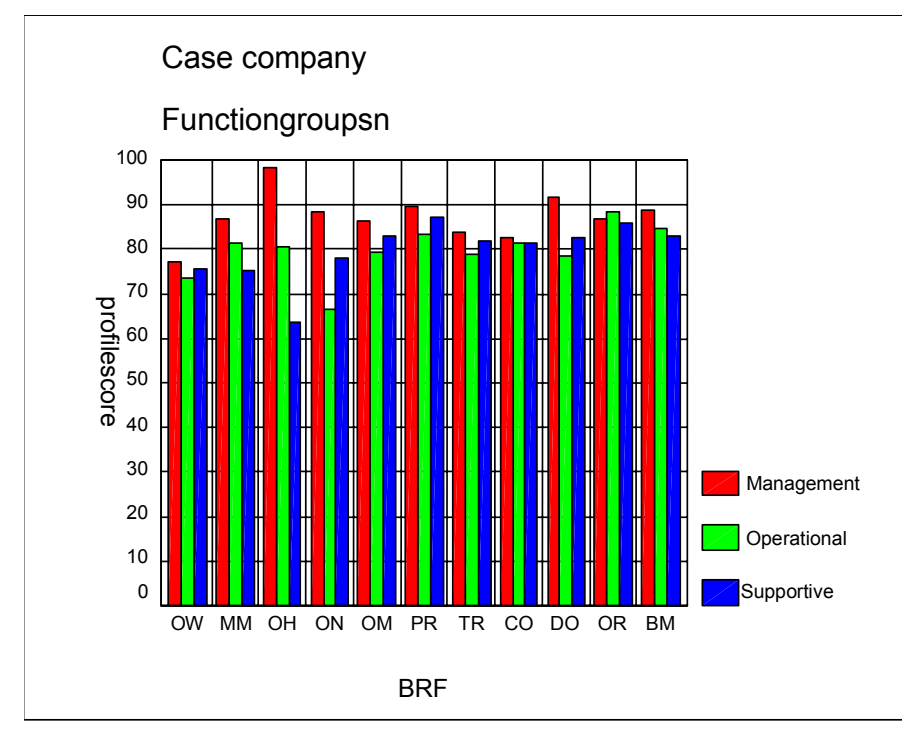

Figure 3: Profile of the case company on the Tripod questionnaire (Abbr. In Dutch) 


\subsection{Integration in the SQF}

The Safety Quality Factor (SQF) should (at least) give an indication of the quality of organisational safety within a company and of the degree in which it influences the independent protection layers or initiating events of the identified scenario's. At this stage we have not been searching for a real quantitative method but we have used the general outcomes of the performed surveys to calculate the quality of the organisational safety. The safety quality factor is based on the OHSAS quick scan and the Tripod survey. Because the Safety culture quick scan has been predominantly a qualitative assessment we have used this instrument as a validation for the semi-quantitative outcome. This leads to a semi-quantitative verdict of the influence this has on the IPL's.

\subsubsection{Calculating the results}

The overall score of the case company on the quick scan OHSAS audit has been 3,9 on a scale from 1 to 5 . Because we are interested in a score which is comparable to the Tripod score (1-10) the OHSAS score has been transferred to this scale: $a x+b=$ y. This gives 7,5 as a result. To determine the relevance of this score, we consider the following scale division for an OHSAS-audit based on experiences with the use of the OHSAS audit as a norm for safety management systems ${ }^{1}$ :

- $10 \%$ of the companies score between $9-10$

- $60 \%$ of the companies score between $5-9$

- $30 \%$ of the companies score between $1-5$.

This leads to a score division of: Good: $8-10$ (best $25 \%$ of the companies); Pass: $6,3-8$; Moderate: $4,3-6,3$; Bad: $1-4,3$. So the score of the case company is in the scale 'pass'.

The overall score of the case company on the Tripod-survey was 81 on a scale of 1 to 100 . A comparison with the results of companies that underwent the same survey reveals the following ${ }^{2}$ :

- $1 \%$ of the companies score between 90-100

- $66 \%$ of the companies score between $70-90$

- $33 \%$ of the companies score between $50-70$.

This leads to a score division of: Good: $82,7-100$ (best $25 \%$ of the companies); Pass: 75,2 - 82,7; Moderate: 65,2 - 75,2; Bad: $50-65,2$. So the score of the case company is in the scale 'pass'.

\subsubsection{Determining the $S Q F$}

The SQF is determined by combining the three techniques. Because organisational failures are often very important contributors to the causes of accidents [8] the four-

\footnotetext{
${ }^{1}$ This scale divison is based on several quick scan OHSAS audits performed between 1998 - 2003.

2 This scale division is based on more than 100 Tripod Delta surveys performed between 1996 and 2003 (information of Tripod International BV).
} 
point scale is divided in two negative, one neutral and one positive result. This is to make sure that any upgrade of the quantitative calculation on a risk assessment is only allowed when organisational safety is really good. The SQF is determined in the following manner.

\begin{tabular}{|l|l|l|l|l|l|}
\hline Survey & $\begin{array}{l}\text { Bad } \\
(--)\end{array}$ & $\begin{array}{l}\text { Moderate } \\
(-)\end{array}$ & $\begin{array}{l}\text { Pass } \\
(0)\end{array}$ & $\begin{array}{l}\text { Good } \\
(+)\end{array}$ & $\begin{array}{l}\text { Final } \\
\text { conclusion }\end{array}$ \\
\hline - OHSAS 18001 & & & $\mathrm{x}$ & & $\begin{array}{l}\text { The SQF is } \\
\text { considered to } \\
\text { be ' } 0^{\prime} .\end{array}$ \\
\hline - Tripod meting & & & $\mathrm{x}$ & & \\
\cline { 1 - 3 } - Quick scan safety culture & $\begin{array}{l}\text { Some ineffective safety cultural } \\
\text { findings }\end{array}$ \\
\hline
\end{tabular}

Table 4 Conclusion of organisational safety of the case company

\subsubsection{SQF and LOPA}

The safety quality factor is used to correct the results of the LOPA analysis. This may result in higher frequencies of failure of protection layers or initiating events resulting in higher scenario frequencies. For example, if we find serious problems in the way safety is organised, the failure frequency of an Independent Layer of Protection will be increased. In this case the company studied had a safety quality factor of ' 0 ', which means that for any range of numbers to be used in the calculations of the scenario's the middle or mean number has to be used.

Apart from the calculation of the SQF and the integration of its result in the LOPA analysis, we drew some general conclusions based on the three different analyses. In this case we concluded that in this company many safety measures are present, in a general sense. However, when looking into more detail we found that the company lacks precision and is nonchalant in implementing and controlling procedures and regulations. This leads to several occurrences of non-safe behaviour or dangerous situations.

\section{Conclusion and discussion}

The main conclusion of the project is that a fruitful combination of technical and organisational safety can be achieved by use of a Safety Quality Factor (SQF). The SQF is determined by quantifying the results of organisational safety studies, and using the result to adept the findings of the technical safety analyses, as summarised in LOPA scenario's.

With respect to the three organisational safety methods it can be concluded that it is possible to give both a (semi-) quantitative and a qualitative judgement on the organisational safety of a company.

A combination of three organisational analysis methods discloses additional facts and evidence and it leads to more opportunities to improve safety performance. At the same time the three different methods all revealed weak spots in the organisation 
with different focus, and can therefore be considered good internal validation tools. In the end the case company has got a lot of relevant information about the process safety and the quality of the safety organisation.

The next step will be development of a management tool to increase rational decision-making based on the LOPA-analysis and the organisational safety findings. In such a tool the cost-effectiveness of proposed safety measures (aimed at closing safety gaps) will have to be assessed. Also, safety performance indicators, to be used in a Balanced Score Card, will be developed, together with management. We will present these results at the congress.

\section{References}

1) Sonnemans PJM, Körvers PMW, Brombacher AC, Van Beek PC and Reinders JEA. Accidents, often the result of an 'Uncontrolled Business process'- a study in the (Dutch) Chemical Industry. Quality and Reliability Engineering International 2003 (in press).

2) Hale AR, Guldenmund FW, Smit K \& Bellamy L. Modification of technical risk assessment with management weighting factors. Safety and reliability - ESREL 1998.

3) Bellamy L. I-RISK, integrating management and technical systems. OECD workshop on audits and inspections 2001

4) Körvers PMW, Schaafsma J, Sonnemans PJM. Invest in safety or invest in production: a dilemma? Making process safety pay, the business case. Center for Chemical Process Safety, International Conference and Workshop. 2001

5) Gort J, Zwetsloot G.I.J.M., Lemkowitz S, Steijger N. and Moonen C. Ensuring safety during manpower changes in the chemical process industry. Symposium on Human error, London. 2003.

6) Layer of Protection Analysis, simplified process risk management. CCPS of the American Institute of Chemical Engineers, 2001.

7) OHSAS 18001, Occupational health \& safety management systems - specification. ICS code 13.100. NEN, 2000.

8) Groeneweg, J. Controlling the controllable. Preventing business upsets, fifth edition. Center for Safety research, Unit of Cognitive Pychology. Global Safety Group, 2002. ISBN 90-6695-140-0. 